\title{
The relationship between insomnia and depressive symptoms: genuine or artifact?
}

\author{
This article was published in the following Dove Press journal: \\ Neuropsychiatric Disease and Treatment \\ II February 20II \\ Number of times this article has been viewed
}

Fadia Isaac

Kenneth Mark Greenwood

School of Health Sciences, RMIT University, Bundoora, Victoria, Australia
Correspondence: Fadia Isaac

School of Health Sciences, RMIT

University, PO Box 7I, Bundoora,

Victoria 3083, Australia

Tel +6I 399257524

Fax +6I 399256539

Emails3222985@student.rmit.edu.au
Background: Somatic symptom overlap between depression and insomnia has emerged as a major concern. Self-report measures such as the Beck Depression Inventory Second Edition (BDI-II) include somatic symptoms related to depression that are also present in the research diagnostic criteria for insomnia. This study aimed firstly to examine the relationship between the cognitive and somatic factors of the BDI-II and global scores on the Pittsburgh Sleep Quality Index (PSQI) in individuals presenting for insomnia treatment and secondly to examine whether treating insomnia in depressed individuals with insomnia will lead to a reduction in their depressive symptoms and whether this reduction is related to a decrease in the somatic or cognitive factors of depressive symptoms.

Methods: A total of 379 individuals (133 males and 246 females), with a mean (M) age of 49.95 (standard deviation $[\mathrm{SD}]=14.15$ ) years, were used to address the first aim. To address the second aim, a total of 64 participants ( 27 males and 37 females) with both insomnia and depressive symptoms were treated for their insomnia. Their ages ranged between 22 and $87(\mathrm{M}=50.97, \mathrm{SD}=15.13)$ years

Results: A significant relationship was found between both the cognitive and somatic factors of the BDI-II and global scores on the PSQI. Furthermore, although results in this study are only suggestive, they lend support to the idea that the relationship between insomnia and depression is not due to somatic symptom overlap. Results may also support the hypothesis that insomnia is primary to the presentation of depressive symptoms.

Conclusion: Clinicians and health care providers could initially treat insomnia in individuals suffering from insomnia who also experience depressive symptoms, as this will not only remit insomnia but also abate the accompanying depressive symptoms.

Keywords: cognitive depressive symptoms, somatic depressive symptoms, symptom overlap

\section{Introduction}

Insomnia is the most common sleep problem, and its consequences are serious. ${ }^{1-5}$ Severe insomnia is associated with increased psychiatric illness, with depression being the most common. ${ }^{6}$ Investigating the relationship between depression and insomnia is important due to their frequent co-occurrence. ${ }^{6-8} \mathrm{~A}$ better understanding of the relationship between the two conditions could aid clinicians in making informed decisions about the best treatment approaches for comorbid insomnia and depression. ${ }^{9}$

The disorders share a complex theoretical and clinical relationship. ${ }^{7}$ It is estimated that $60 \%$ of adults meeting criteria for major depressive disorders (MDDs) complain of insomnia, and approximately $10 \%-20 \%$ of individuals diagnosed with insomnia meet criteria for MDDs. ${ }^{10}$ The direction of the relationship between insomnia 
and depression has emerged as a major concern in the literature. ${ }^{11-14}$ Although some suggest that depression is the largest and most consistent risk factor for chronic insomnia, ${ }^{13,14}$ others argue that insomnia is a risk factor for the development of depression. ${ }^{12}$ The Diagnostic and Statistical Manual of Mental Disorders Fourth Edition, Text Revision (DSMIV-TR) classifies insomnia into two categories: primary insomnia, associated with sleep onset and/or maintenance problems, and secondary insomnia, associated with medical or mental health conditions. ${ }^{15}$ This classification implies that insomnia experienced in combination with psychological disorders such as depression is secondary to (caused by) the psychological disorder.

Interestingly, much research suggests otherwise. Following an extensive review of the literature, Harvey concluded that insomnia precedes the development of depression. ${ }^{16}$ More recent findings are in line with Harvey's conclusion. In a sample of 1014 youths, Johnson et al found that after adjusting for gender, race, and prior anxiety disorders, insomnia occurred first in $69 \%$ of cases with comorbid insomnia and depression. ${ }^{12}$ On the other hand, depression was not found to be associated with the onset of insomnia. Similarly, in a longitudinal study in Switzerland, Buysse et al found that pure and comorbid insomnia was associated with future episodes of major depression or pure insomnia. ${ }^{17}$ However, pure depression was less strongly related to future episodes of comorbid insomnia and depression, pure depression, or pure insomnia. ${ }^{17}$

A better understanding of the causal nature of the relationship between insomnia and depression may be obtained by treating one condition and monitoring changes in the other. Research in this area has been limited. Morawetz found that $70 \%$ of individuals with insomnia and depression who received treatment for their insomnia experienced a decrease in their depression. Individuals who did not experience improvement in their insomnia did not show improvement in their depression. Morawetz concluded that these results were suggestive of depression being secondary to insomnia. ${ }^{18}$ Although different methods were utilized to treat insomnia, other researchers have come to a similar conclusion. ${ }^{19,20}$

Research treating depression and observing insomnia has been very limited. Carney et al reported improvements in insomnia following the treatment of depression. ${ }^{21}$

A second major concern is associated with the overlap of some somatic symptoms present in both conditions (concentration difficulties, reduced sleep, and fatigue). ${ }^{21}$ More specifically, self-report measures assessing the severity of depression, such as the Beck Depression Inventory
Second Edition (BDI-II), ${ }^{23}$ include somatic symptoms related to depression that are also present in the research diagnostic criteria for insomnia, eg, insomnia, irritability, fatigue, concentration difficulties, motivation, and mood disturbance. ${ }^{24}$ This overlap makes it difficult to distinguish whether the elevated total scores reported on measures of depression such as the BDI-II in those with sleep problems reflect genuine depression, or whether the elevated scores are due to the overlap of the somatic symptoms common to both conditions. There is very little research addressing this issue.

One study addressed this issue by examining the correlation of each item on the BDI-II with measures of sleep. Utilizing the Duke Structured Interview for Sleep Disorders (DSISD), ${ }^{25}$ participants were screened for the presence of insomnia; they were also evaluated for the presence of MDDs using the structured clinical interview for the DSM-IV-TR. ${ }^{26}$ Carney et al allocated participants into two groups (insomnia with or without MDD). Following administration of the BDI-II, they found that the group with comorbid insomnia and depression scored significantly higher than the individuals with pure insomnia on 13 of the 21 items of the BDI-II. These items were sadness, crying, past failure, self-dislike, pessimism, anhedonia, guilt, agitation, indecision, worthlessness, energy, hypersomnia, and loss of appetite. ${ }^{26}$ However, the two groups did not differ on eight items (insomnia, irritability, suicidal thoughts, decreased concentration, fatigue, libido, thoughts of punishment, and critical thoughts of the self), indicating some degree of symptom overlap.

Studies reported in this paper have examined improvements in the level of depression following the treatment of insomnia without considering potential differential response in the cognitive and somatic aspects of depression. ${ }^{18-20}$ In fact, no study has examined whether improvement in individuals' mood states were due to the reduced scores on the somatic or cognitive aspects of their depressive symptoms.

The present study aimed to examine the relationship between both the cognitive and somatic factors of the BDI-II and insomnia. This study also aimed to explore whether an initial treatment of insomnia in individuals presenting with both insomnia and depressive symptoms who volunteered to take part in an intervention for insomnia would result in a reduction in depressive symptoms and whether this decrease would be due to a reduction in the somatic or cognitive factors or both.

\section{Method}

\section{Participants}

A total of 379 individuals (133 males and 246 females) who were concerned about their sleep and volunteered to take 
part in an intervention for insomnia participated in this study. Their ages ranged between 18 and 90 (mean $[\mathrm{M}]=49.95$, standard deviation $[\mathrm{SD}]=14.15$ ) years.

Of the 379 participants, a subsample of individuals who met the Lichstein et $\mathrm{al}^{27}$ criteria for insomnia, who scored greater than 12 on the BDI-II prior to intervention and who also completed the intervention and provided postintervention data on the Pittsburgh Sleep Quality Index (PSQI) and the BDI-II, were selected. A total of 64 participants (27 males and 37 females) were identified. Their ages ranged between 22 and $87(\mathrm{M}=50.97, \mathrm{SD}=15.13)$ years.

\section{Measures}

\section{The Pittsburgh Sleep Quality Index (PSQI) $^{28}$}

The PSQI is a self-rated questionnaire assessing the individual's perception of the quality of his/her sleep over the preceding month. The PSQI consists of 19 questions that are grouped into seven components reflecting different aspects of sleep. Each question is scored on a scale ranging from 0 to 3, with 0 indicating the absence of sleep complaints and 3 indicating the most severe complaints. The seven components are then added to form the global score of the PSQI, ranging from 0 to 21 , with higher scores indicating worse sleep quality. ${ }^{28}$ Scores higher than 5 on the global score are a reflection of severe sleep problems. ${ }^{29}$ The PSQI is a reliable measure, with test-retest correlation of 0.87 for the global scores and between 0.53 and 0.88 for the component scores for patients with primary insomnia. ${ }^{30}$ Good evidence of validity has been reported. ${ }^{31,32}$

\section{The Beck Depression Inventory Second Edition (BDI-II) $)^{23}$}

The BDI-II measures the severity of depressive symptoms in accordance with the DSM-IV. The BDI-II is a 21-item self-report measure, with each item rated on a 4-point ordinal response scale ranging from 0 , which indicates the absence of symptoms, to 3 , indicating the most severe symptoms. The total BDI-II score is the sum of the 21 items and ranges from 0 to 63 , with higher scores indicating more severe depressive symptoms. Scores ranging between 0 and 13 reflect an absence of, or minimal, depressive symptoms; scores between 14 and 19 reflect mild depressive symptoms; between 20 and 28 reflect moderate depressive symptoms; and between 29 and 63 reflect severe depressive symptoms. ${ }^{23}$ Beck et al suggest that the instrument measures two factors: the somatic-affective factor/subscale, which includes 12 of the 21 items (loss of pleasure, crying, agitation, loss of interest, indecisiveness, loss of energy, changes in sleeping patterns, irritability, changes in appetite, concentration difficulties, tiredness or fatigue, and loss of interest in sex), and the cognitive factor/subscale, which includes nine items (sadness, pessimism, past failure, guilt, punishment feelings, self-dislike, self-criticalness, suicidal thoughts, and worthlessness). ${ }^{23}$ The BDI-II has an internal consistency coefficient $\alpha$ of 0.93 and a test-retest reliability of $0.93 .^{23}$ Moreover, the BDI-II has been found to be a valid scale for measuring depression in chronic pain patients ${ }^{33}$ and traumatic brain injury, ${ }^{34}$ and, more importantly, the BDI-II has high accuracy in discriminating between individuals diagnosed with insomnia and depression and those diagnosed with insomnia without depression. ${ }^{26}$

Although a formal clinical interview for depression might have been preferred, it was not feasible, as the primary focus of the main study was concerned with insomnia. Readers should note that this paper reports on depressive symptoms and not clinically diagnosed depression.

\section{Procedure}

Following the approval of La Trobe University, Melbourne, Australia, participants were recruited through advertisements in newspapers, notices in community health centers, and through word of mouth.

A package of questionnaires, including the PSQI and the BDI-II, was sent to all prospective participants. To be eligible to participate in the study, individuals needed to meet the criteria for insomnia specified by Lichstein et al, ${ }^{27}$ ie, they had to have a self-reported sleep onset latency of at least 30 minutes or be awake for at least 30 minutes after sleep onset at least 3 nights per week and had experienced sleep difficulties for at least 6 months. Furthermore, due to ethical concerns, participants were included in the study only if they scored below 29 on the BDI-II. Informed consent was obtained from all selected participants. Participants were assured of the confidentiality of the information and the right to withdraw from the study at any stage.

The intervention involved three treatment groups: a therapist-led group, a group who received a self-help manual, and a group who received a self-help manual and weekly telephone calls from a therapist. The content that all participants were exposed to was as similar as possible and based on the treatment manual published by Morin and Espie. ${ }^{4}$ Those in the self-help groups received a manual that was based on the one published by Morin and Espie in 2003. The treatment manual comprised six modules. Each module provided information and strategies for participants to implement to 
improve their sleep. The first module predominantly focused on environmental factors (eg, noise, temperature) that affect people's sleep. Module 2 examined how poor sleep hygiene (a set of behaviors that are known to influence sleep, eg, vigorous exercise too close to bedtime or nicotine or caffeine) can impact on sleep quality and quantity. The third module focused on six behavioral strategies for good sleep, collectively known as stimulus control. Module 4 described relaxation exercises for participants to incorporate into their prebedtime routine. The fifth module addressed cognitive factors that might be contributing to a long sleep onset or preventing people from resuming sleep if they wake during the night. The strategies described include, but are not limited to, prioritizing activities, problem solving, and assertiveness. The final module utilized the principles of relapse prevention and encouraged participants to review their progress in relation to the goals they originally set for themselves. Those in the therapist-led group were exposed to this content by an experienced therapist in weekly 60-minute sessions conducted over a 6-week period. Those in the self-help groups were asked to complete one module each week over the same period of time. For the purposes of this study, data from the three groups were combined, as the differences in the outcome between the three groups were not significant. Thus, all participants received an intervention using the commonly accepted cognitive behavioral treatment approach for insomnia.

\section{Results}

Prior to intervention, a significant positive correlation was found between global scores on the PSQI $(\mathrm{M}=13.36$, $\mathrm{SD}=3.27)$ and total scores on the BDI-II $(\mathrm{M}=14.55$, $\mathrm{SD}=10.37), r(\mathrm{~N}=379)=0.41, P<0.001, R^{2}=0.17,95 \%$ confidence interval $(0.32,0.49)$. To further investigate whether this relationship differed between the somatic and cognitive factors of the BDI-II, a significant positive relationship was found between global scores on the PSQI and the cognitive factor of the BDI-II $(\mathrm{M}=9.92, \mathrm{SD}=6.28)$, $r(\mathrm{~N}=379)=0.41, P<0.001, R^{2}=0.17(0.32,0.49)$ and also with the somatic factor of the BDI-II $(\mathrm{M}=4.61$, $\mathrm{SD}=4.90), r(\mathrm{~N}=379)=0.34, P<0.001, R^{2}=0.12(0.25,0.43)$. Although a formal test of the difference between the two correlation coefficients using Steiger's $Z^{35}$ failed to reach significance $(Z=1.12, P=.26)$, the relationship for the somatic factor was relatively weaker in comparison with that observed between the PSQI and the cognitive factor. If the relationship between insomnia and depression was due to overlap of somatic items on both instruments, one would have expected the correlation with the somatic factor of the BDI-II and the PSQI to be superior to that with the cognitive factor. All three correlations reported were of moderate magnitude. ${ }^{36}$ Figure 1 shows a matrix of scatterplots among the variables prior to intervention.

To investigate how the intervention impacted on the cognitive and somatic factors of depression, paired-sample $t$-tests were used. The mean score for the somatic factor after intervention ( $\mathrm{M}=4.24, \mathrm{SD}=3.42$ ) was significantly lower than the mean score before intervention $(\mathrm{M}=6.15$, $\mathrm{SD}=3.08), t(65)=5.48, P<0.001, d=0.67(0.40,0.94)$. For the cognitive factor, the mean score after intervention $(\mathrm{M}=8.09, \mathrm{SD}=4.82)$ was also significantly lower than the mean score before intervention $(\mathrm{M}=12.41, \mathrm{SD}=3.54)$, $t(65)=7.23, P<0.001, d=0.89$ (0.60, 1.17). Although both effect sizes were in the moderate range, ${ }^{36}$ Steiger's $Z^{35}$ revealed that these effect sizes were significantly different $(Z=2.81, P=0.004)$, with a larger effect observed on the cognitive factor. The mean of the BDI-II total scores after intervention $(\mathrm{M}=12.51, \mathrm{SD}=7.59)$ was significantly lower than the mean scores of the BDI-II before intervention $(\mathrm{M}=18.63, \mathrm{SD}=4.54, t(64)=7.59, P<0.001, d=0.94$ $(0.65,1.23)$. The effect size was moderate. ${ }^{36}$ Figure 2 shows the means for the PSQI global scores, the BDI-II total scores, and the somatic and cognitive factors prior to and following the treatment of insomnia.

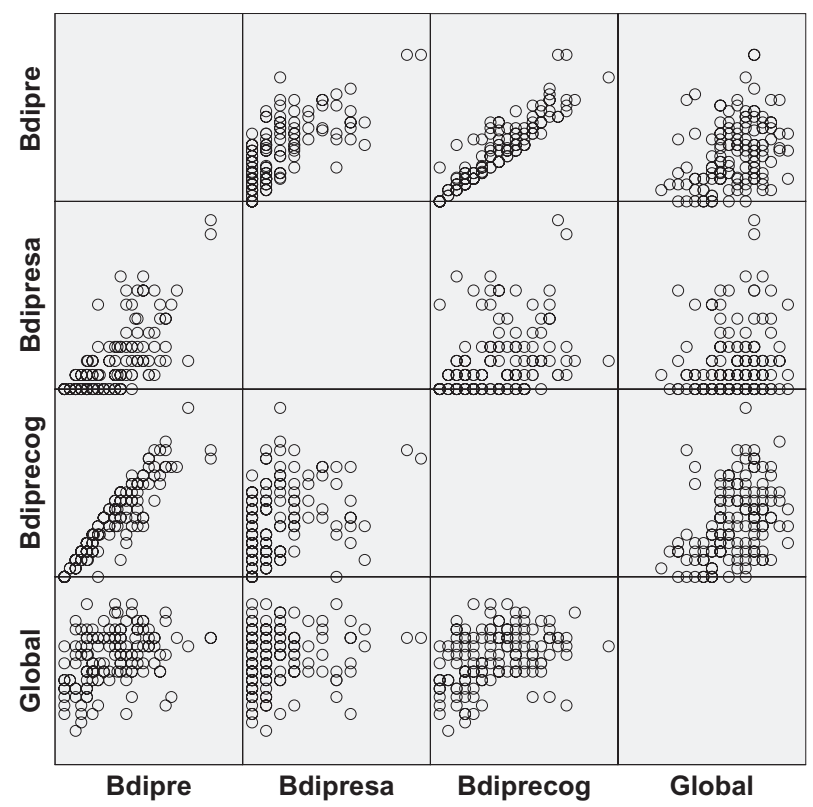

Figure I Matrix scatterplot showing Pittsburgh Sleep Quality Index (PSQI) global scores prior to intervention (global), the Beck Depression Inventory Second Edition (BDI-II) prior to intervention (bdipre), the cognitive factor score on the BDI-II prior to intervention (bdiprecog), and the somatic factor score on the BDI-II prior to intervention (bdipresa). 


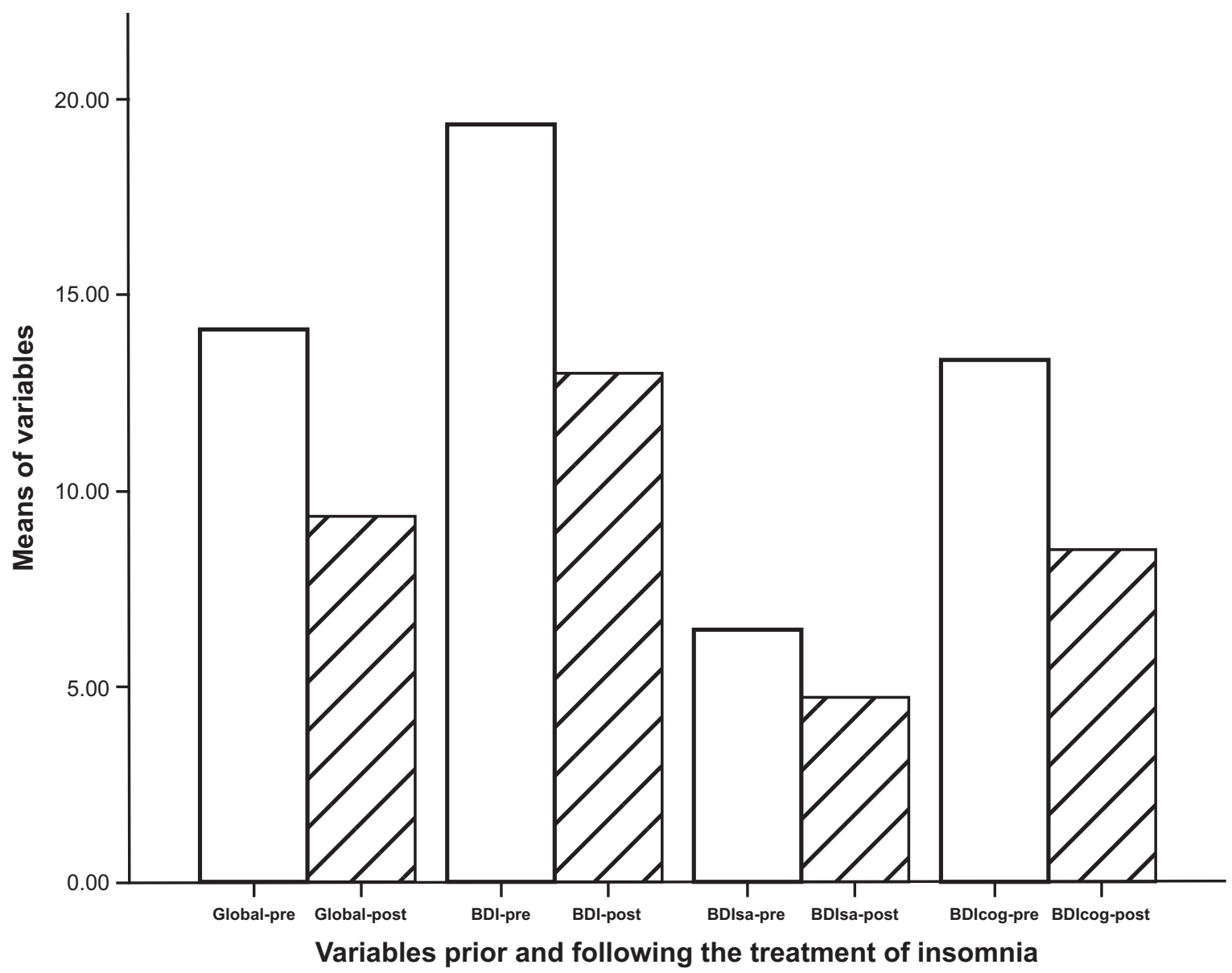

Figure 2 Means for the Beck Depression Inventory Second Edition (BDI-II) total scores, the cognitive factor score on the BDI-II (BDIcog), the somatic factor score on the BDI-II (BDIsa), and the Pittsburgh Sleep Quality Index global scores (Global-Pre and Global-Post) prior to and following the treatment of insomnia.

A paired-sample $t$-test showed that the mean global PSQI score after intervention $(\mathrm{M}=9.28, \mathrm{SD}=3.37)$ was significantly lower than the mean score of the PSQI before intervention $(\mathrm{M}=14.14, \mathrm{SD}=2.56), t(63)=12.36, P<0.001, d=1.55$ $(1.18,1.91)$. The effect size was large. ${ }^{36}$

The mean of the BDI-II total scores after intervention $(\mathrm{M}=12.51, \mathrm{SD}=7.59)$ was significantly lower than the mean scores of the BDI-II before intervention $(\mathrm{M}=18.63$, $\mathrm{SD}=4.54, t(64)=7.59, P<0.001, d=0.94(0.65,1.23)$. The effect size was moderate. ${ }^{36}$

A Pearson correlation revealed that the change in global scores on the PSQI was significantly positively correlated with the change of scores on the BDI-II total scores, $r(\mathrm{~N}=63)=0.43, P<0.001, R^{2}=0.18(0.20,0.61)$. There was also a positive significant relationship between the change in global scores and the change in cognitive scores on the BDI-II, $r(\mathrm{~N}=63)=0.44, P<0.001, R^{2}=0.19$ $(0.21,0.62)$. However, there was no significant relationship between global scores and the change in somatic scores on the BDI-II, $r(\mathrm{~N}=63)=0.23, P=0.06, R^{2}=0.05(0.01,0.45)$.

\section{Discussion}

Prior to intervention, a positive relationship was found between insomnia, as measured by the PSQI, and depressive symptoms, as measured by the BDI-II, and for both the somatic and cognitive factors. Although there was no significant difference between the cognitive and somatic factors and the relationship with the PSQI, this relationship accounted for $17 \%$ of the shared variance with the cognitive factor in comparison with only $12 \%$ of the shared variance with the somatic factor. Even though the findings are not statistically significant and are only suggestive, this finding is surprising and contradicts the concerns raised in the literature ${ }^{22}$ that the relationship between insomnia and depressive symptoms is due to the somatic symptom overlap in both conditions. If this were the case, one would expect the correlation to be much higher with the somatic factor than with the cognitive factor. This supports Carney et al's findings, which suggest that the relationship between insomnia and depressive symptoms is genuine and not due to measurement artifact. $^{26}$ 
Those receiving treatment for their insomnia also showed a decrease in their depressive symptoms. Following intervention, PSQI global scores were significantly reduced, and the magnitude of this change was large. Furthermore, total scores on the BDI-II were also significantly reduced. However, the magnitude of the decrease in depressive symptoms was moderate. This is possibly due to the fact that participants with severe depressive symptoms were excluded from the study. A different magnitude of effect for depressive symptoms may have been observed had individuals with severe depressive symptoms been included. Future research ought to investigate this possibility. This result is similar to previous work that showed that exclusive treatment of insomnia in individuals experiencing both depression and insomnia resulted in decreases in depression. ${ }^{18-20}$

The pattern of change differed for the somatic and cognitive factors on the BDI-II. Interestingly, the cognitive factor was reduced more in comparison with the somatic factor. This finding could be explained by the results reported earlier, which found a stronger correlation between cognitive scores and the PSQI scores. Although both factors showed a moderate reduction, the difference between the two effect sizes was significant.

Those reporting the most improved sleep showed the most effective change on the total scores of the BDI-II. However, the relationship for change of scores between insomnia and depressive symptoms was found only for the cognitive factor accounting for $23 \%$ of the shared variance and not found for the somatic factor accounting for a small $0.5 \%$ of the shared variance. These findings strengthen the conclusion made earlier in this study, that the relationship between insomnia and depressive symptoms is a genuine relationship and is not due to the measurement artifact of shared somatic items in both instruments.

An implication of this study is that individuals with both insomnia and depressive symptoms may benefit from an initial treatment for their insomnia, as this not only will improve their sleeping but also may improve their mood state. Following improvements on their insomnia and possibly depressive symptoms, therapy may be directed to treat any residual depression.

This study is not without its limitations. Although a clinical interview for depression might have been preferred, it was not feasible for this study. The small sample size of 64 participants must not be overlooked, and future research should target a larger sample size to confirm the current findings. To select a sample of individuals with sleep problems who were also experiencing some degree of depression, the present study required a score of more than 12 on the BDI-II following the descriptive labels provided by Beck et al to include those with mild and moderate depression. ${ }^{22}$ However, Beck et al proposed a cutoff of 17 as a good criterion for clinical research, and this was used by Carney et al. ${ }^{26}$ The use of a higher cutoff was precluded in this study, as the sample size would have been too small to allow for powerful analyses. Furthermore, being the first study to explore somatic and cognitive symptoms and their relationship with insomnia, there was a need to include individuals experiencing mild depressive symptoms. Future research should focus more on this issue of the appropriate level of depressive symptoms to include in studies of this kind. As reported earlier, the PSQI has seven components that explore various aspects of sleep quality, and future research could examine the relationship between these components and somatic and cognitive symptoms.

In conclusion, this is the first study to explore the relationship between insomnia and depressive symptoms, and specifically the somatic and cognitive factors of the BDI-II. The study revealed some interesting and unexpected findings. Taken together, the findings from the current study suggest that there is a genuine relationship between insomnia and depressive symptoms and that this relationship is not due to the somatic symptom overlap seen in both conditions. Furthermore, following the treatment of insomnia, depressive symptoms were reduced. More importantly, the cognitive factor experienced the most reduction in comparison with the somatic factor, which experienced no reduction. The current findings may lend support to the hypothesis that depression is secondary to insomnia.

\section{References}

1. Ambrogetti A, Hensley MJ, Olson LG. Sleep Disorders: a Clinical Textbook. London: Quay Books; 2006.

2. Caliyurt O, Guducu F. Partial sleep deprivation therapy combined with sertraline affects subjective sleep quality in major depressive disorder. J Affect Disord. 2005;88(1):75-78.

3. Brunello N, Armitage R, Feinberg I, et al. Depression and sleep disorders: clinical relevance, economic burden and pharmacological treatment. Neuropsychobiology. 2000;42(3):107-119.

4. Morin CM, Espie CA. Insomnia: a Clinical Guide to Assessment and Treatment. New York, NY: Kluwer Academic; 2003.

5. Naitoh P, Kelly TL, Englund CE. Health effects of sleep deprivation. Occup Med. 1990;5(2):79-89.

6. Sarsour K, Morin CM, Foley K, et al. Association of insomnia severity and comorbid medical and psychiatric disorders in a health plan-based sample: insomnia severity and comorbidities. Sleep Medicine. 2010; 11(1):69-74.

7. Nowell PD, Buysse DJ. Treatment of insomnia in patients with mood disorders. Depress Anxiety. 2001;14(1):7-18.

8. Taylor DJ. Commentary on Buysse et al. Prevalence, course, and comorbidity of insomnia and depression in young adults. Sleep. 2008;31(4):473-480. 
9. Riemann D. Insomnia and comorbid psychiatric disorders. Sleep Medicine. 2008;8(4):15-20.

10. Ohayon MM. Insomnia: a ticking clock for depression? J Psychiatr Res. 2007;41(11):893-899.

11. Benca RM, Peterson MJ. Insomnia and depression. Sleep Medicine. 2008;Suppl 1:S3-S9.

12. Johnson EO, Roth T, Breslau N. The association of insomnia with anxiety disorders and depression: exploration of the direction of risk. J Psychiatr Res. 2006;40(8):700-708.

13. Katz DA, McHorney CA. Clinical correlates of insomnia in patients with chronic illness. Arch Intern Med. 1998;158(10):1099-1107.

14. Ohayon MM, Caulet M, Lemoine P. Comorbidity of mental and insomnia disorders in the general population. Compr Psychiatry. 1998;39(4): 185-197.

15. American Psychiatric Association. Diagnostic and Statistical Manual of Mental Disorders. 4th ed. Washington, DC: American Psychological Association; 2000.

16. Harvey AG. Insomnia: symptom or diagnosis? Clin Psychol Rev. 2001; 21(7):1037-1059.

17. Buysse D, Angst J, Gamma A, et al. Prevalence, course, and comorbidity of insomnia and depression in young adults. Sleep. 2008;31(4): 473-480.

18. Morawetz D. Insomnia and depression: which comes first? J Sleep Res. 2003;5(2):77-81.

19. Casper RC, Katz MM, Bowden CL, et al. The pattern of physical symptom changes in major depressive disorder following treatment with amitriptyline or imipramine. J Affect Disord. 1994;31(3): $151-164$.

20. Jacobs GD, Benson H, Friedman R. Home-based central nervous system assessment of a multifactor behavioural intervention for chronic sleeponset insomnia. Behaviour Therapy. 1993;24(1):159-174.

21. Carney CE, Segal ZV, Edinger JD, Krystal AD. A comparison of rates of residual insomnia symptoms following pharmacotherapy or cognitive-behavioral therapy for major depressive disorder. J Clin Psychiatry. 2007;68(2):254-260.

22. Moul DE, Nofzinger EA, Pilkonis PA, et al. Symptom reports in severe chronic insomnia. Sleep. 2002;25(5):548-558.

23. Beck AT, Steer RA, Brown GK. Beck Depression Inventory BDI-II Manual. 2nd ed. Orlando, FL: Psychological Corporation; 1996.
24. Edinger JD, Bonnet M, Bootzin RR, et al. Derivation of research diagnostic criteria for insomnia: report on an American Academy of Sleep Medicine work group. Sleep. 2004;27(8):1567-1596.

25. American Academy of Sleep Medicine. International classifications of sleep disorders-second edition (ICSD-2). In: Hauri PJ, editor. International Classification of Sleep Disorders. Chicago, IL: Steering Committee; 2005:75-150.

26. Carney CE, Ulmer C, Edinger JD, et al. Assessing depression symptoms in those with insomnia: an examination of the Beck Depression Inventory Second Edition (BDI-II). J Psychiatr Res. 2009;43(5):576-582.

27. Lichstein KL, Durrence HH, Taylor DJ, et al. Quantitative criteria for insomnia. Behav Res Ther. 2003;41:427-445.

28. Buysse DJ, Reynolds CF, Monk TH, et al. The Pittsburgh Sleep Quality Index: a new instrument for psychiatric practice and research. J Psychiatr Res. 1989;28(2):193-213.

29. Mimeault V, Morin CM. Self-help treatment for insomnia: bibliotherapy with and without professional guidance. J Consult Clin Psychol. 1999; 67(4):511-519.

30. Backhaus J, Junghanns K, Broocks A, et al. Test-retest reliability and validity of the Pittsburgh Sleep Quality Index in primary insomnia. J Psychosom Res. 2002;53(3):737-740.

31. Germain A, Hall M, Krakow B, et al. A brief sleep scale for posttraumatic stress disorder: Pittsburgh Sleep Quality Index addendum for PTSD. J Anxiety Disord. 2005;19(2):233-244.

32. Beck S, Schwartz AL, Towsley G, et al. Psychometric evaluation of the Pittsburgh Sleep Quality Index in cancer patients. J Pain Symptom Manage. 2004;27(2):140-148.

33. Poole H, Bramwell R, Murphy P. Factor structure of the Beck Depression Inventory-II, patients with chronic pain. Clin J Pain. 2006;22(9): 790-798.

34. Rowland SM, Lam CS, Leahy B. Use of the Beck Depression Inventory-II (BDI-II) with persons with traumatic brain injury: analysis of factorial structure. Brain Inj. 2005;19(2):77-83.

35. Meng XL, Rosenthal R, Rubin DB. Comparing correlated correlation coefficients. Psychol Bull. 19992;111(1):172-175.

36. Hopkins WG. A new view of statistics. 2009. http://www.sportsci.org/ resourc/stats/index.html. Accessed March 2, 2010.
Neuropsychiatric Disease and Treatment

\section{Publish your work in this journal}

Neuropsychiatric Disease and Treatment is an international, peer-reviewed journal of clinical therapeutics and pharmacology focusing on concise rapid reporting of clinical or pre-clinical studies on a range of neuropsychiatric and neurological disorders. This journal is indexed on PubMed Central, the 'PsycINFO' database and CAS, and is the

\section{Dovepress}

official journal of The International Neuropsychiatric Association (INA). The manuscript management system is completely online and includes a very quick and fair peer-review system, which is all easy to use. Visit http://www.dovepress.com/testimonials.php to read real quotes from published authors. 